http://doi.org/10.15359/ree.14-Ext.3

\title{
El placer de aprender
}

\section{The Pleasure of Learning}

\author{
Luz Emilia Flores Davis ${ }^{l}$ \\ División de Educación Básica del \\ Centro de Investigación y Docencia en Educación (CIDE) \\ Universidad Nacional \\ Heredia, Costa Rica
}

Recibido 22 de marzo de $2010 \bullet$ Aceptado 24 de junio de 2010

\begin{abstract}
Resumen. La evolución de una educación centrada en la enseñanza, a una perspectiva de aprendizaje, constituye un cambio de paradigma, así lo afirma la autora de este artículo, quien invita a asumir el placer de aprender como dinamizador de la construcción de sentidos. Asevera que la educación debe transitar de la repetición acrítica de contenidos, a la generación de aprendizaje placentero, y que la búsqueda de alternativas para una educación que supere el paradigma tradicional, lleva a la necesidad de promover una auténtica participación gozosa.

Palabras clave. Paradigma emergente, pedagogía, aprendizaje, placer construcción, conocimientos.

Abstract. The evolution, from an education centered in teaching to a perspective of learning, constitutes a change of paradigm as stated by the author in this article - who invites us to use the pleasure of learning as a facilitator for the construction of the senses. The author underlines that education must advance from the non-critical repetition of content to the generation of pleasant learning, and that the search for alternatives for an education that overcomes the traditional paradigm leads to the need to promote an authentic learning pleasure.
\end{abstract}

Key words. Rising paradigm, pedagogy, learning, pleasure, construction, knowledge.

\section{Introducción}

El momento apremiante que vivimos, en la actualidad, para la supervivencia de la vida en el planeta y para hacer frente a un mundo que sufre de pobreza e inequidad, nos coloca en una situación límite que urge una transformación social. En este sentido, la educación tiene un desafío decisivo en la búsqueda de caminos alternativos, de opciones para la construcción de un mundo

\footnotetext{
Doctora en Educación con mención en Mediación Pedagógica. Máster en Educación Superior. Catedrática universitaria. Ha ocupado los cargos de Directora de Docencia, Directora de Desarrollo Profesional y Cooperación Universitaria, Presidenta de la Junta de Becas y Coordinadora de la Comisión Técnica de Admisión de la Universidad Nacional. Actualmente, se desempeña como investigadora, extensionista y docente en la División de Educación Básica del Centro de Investigación y Docencia en Educación (CIDE) de la Universidad Nacional, Costa Rica y docente en la Maestría en Danza con mención en Formación Dancística del Centro de Investigación, Docencia y Extensión Artística (CIDEA), Universidad Nacional, Costa Rica. Correo electrónico: luzdeflores@yahoo.com
} 
solidario y, para ello, es vital un cambio de conciencia que viabilice gestar procesos creativos, en vez de asumir posiciones ingenuas que de un modo simplista realizan modificaciones superficiales donde se requieren profundas innovaciones.

Ese compromiso se relaciona con la formación de un tipo de persona y de sociedad que promueva el impulso humano hacia la belleza y el placer, hacia la solidaridad y la responsabilidad; que ame la vida; que entienda que el cuidado es el eje de las relaciones; un tipo de persona y sociedad que no viva con miedo al dolor; donde la creación tenga espacio fecundo y la sensibilidad sea la fuerza para la transformación.

Es indispensable transitar de una sociedad controladora, que utiliza para la dominación, entre otros, los medios masivos de comunicación -que supuestamente propician participación y democracia-, a una nueva forma de organización social donde la colaboración no nazca de la "obediencia debida" propia del sistema patriarcal, sino del placer de participar con libertad, de construir conjuntamente, de implicarse con pasión.

Urgimos una pedagogía de la vida, una pedagogía que busque significados y significar; que permita expresarse desde la propia historia, desde lo más profundo del ser... Una pedagogía que genere vivir con intensidad. Debemos pasar de la repetición acrítica de contenidos, a la búsqueda de sentidos y a la generación de ambientes de aprendizaje placentero. En ese transitar, el placer constituye un vórtice en la vida cotidiana; este permite olvidar el ego y sumergirse en el terreno caótico que hace posible crear, con la propia vida, utilizando, sin miedo, los errores en la búsqueda de horizontes ignorados.

\section{La educación en el paradigma mecanicista}

El paradigma tradicional, mecanicista, ha llevado a la crisis actual en todos los campos y nos ha dominado imponiéndonos una sensación de impotencia; de esa manera nos ha convertido en personas y sociedades pasivas, oprimidas, acostumbradas a relaciones de subordinación que demandan obediencia y se afirman en la negación de la otra o del otro. El tipo de sociedad construida en los últimos siglos ha sido estructurada sobre un modelo de desarrollo que rompe la armonía de la naturaleza y explota la fuerza de trabajo de amplios sectores de la población.

Nos encontramos ante una sociedad caracterizada por la inequidad, en la que apremia una nueva ética promotora de un decrecimiento del consumo de los grupos opulentos para construir una sociedad que garantice el bien común.

La ilusión de infinito, tanto del desarrollo como de los recursos naturales -supuesto de la sociedad moderna- ha llegado a su fin. La crisis de alimentos, el agotamiento del agua y de los combustibles fósiles, unido a la situación de pobreza de extensos sectores de la sociedad, revela el desenlace del desarrollo lineal. El deterioro ecológico es producto del concepto de desarrollo que se ha manejado, y sus consecuencias ni siquiera se miden adecuadamente (Alvarado, 2007). "Todos los recursos son no renovables y el conjunto de actividades humanas, al acelerar el flujo de materiales y energía hacia el sistema económico, refuerza el proceso inevitable de degradación de la base de recursos" (Gómez, 1998, p. 12).

El modelo de desarrollo propio de la sociedad patriarcal ha forjado tanto la crisis ambiental que hoy nos coloca en una situación crítica para la supervivencia de la humanidad y del planeta, como la crisis social originada en la exclusión y el deterioro de las relaciones humanas. Desde la infancia se nos dice qué debemos pensar, qué debemos sentir, y cómo debemos actuar para adaptarnos al aparato económico. Se suscita la homogenización como estrategia de dominación. Entonces, ¿qué sucede con nuestros deseos, con nuestros sentidos, con nuestras pasiones? 
Los mecanismos de poder nos han limitado a vivir en los modelos establecidos por el mismo poder, haciéndonos creer que la seguridad se encuentra en lo conocido.

La visión mecanicista y fragmentaria característica del paradigma dominante del siglo XX tiene su correlativo en la educación. Es así como su abordaje se ha realizado desde las disciplinas científicas, en detrimento de la valoración de la vida cotidiana, de las artes, de la actividad física, y de la persona misma. El uso del lenguaje es revelador en este sentido: se habla de "programas" de cursos, así como de "ejes" del currículo, términos propios de las máquinas. En cuanto a la organización escolar, se mencionan horarios "mosaico", lo cual no deja duda del planteamiento fragmentado de la práctica educativa en el sistema formal.

Se plantea que determinadas materias son "básicas", en detrimento del resto, con lo cual se cercena el desarrollo integral. Para referirse a las personas, se nombra una determinada característica en menoscabo de su integralidad; así se dice, por ejemplo, los "analfabetos" en lugar de "personas que no han aprendido o potenciado aún la lectoescritura convencional"; o "discapacitado" en lugar de "persona con una situación particular".

La educación en el paradigma tradicional no se encuentra vinculada de forma sensible con la vida; se ha encerrado en sí misma y se ha negado a aprender de la vida. En los diversos niveles del sistema educativo, impone una manera de ver el mundo y reprime la alegría, la espontaneidad y la libertad de expresión mediante políticas o directrices que mantienen las condiciones sociales hegemónicas, utilizando mecanismos jerárquicos de control. En ese marco resulta muy conveniente un profesorado que utilice como su mejor arma y escudo la evaluación unidireccional. En este sentido, la educación tradicional tiene una responsabilidad directa en la consolidación de una sociedad cada vez más violenta e incapaz de resolver los conflictos por la vía pacífica y de valorar a la otra o al otro, como una legítima otra u otro.

La evaluación unidireccional ha apoyado la formación de un tipo de persona y de sociedad pasiva, temerosa de las represalias de quien ostenta el poder; que prefiere someterse a arbitrariedades antes que defender su derecho a manifestarse, a discrepar, al verdadero diálogo respetuoso; que es sumisa frente a la autoridad, pero agresiva hacia las subordinadas y los subordinados.

Francisco Gutiérrez plantea que "(...) hay que hacer con placer y ternura en la educación. La educación que tenemos pone en peligro el destino de la humanidad. La educación puede salvar la humanidad, pero no la que tenemos" (Gutiérrez, 2008, p. 1).

Efectivamente, la supervivencia de la humanidad y del planeta requiere de la toma de conciencia de la ilusoria situación de bienestar que presenta el paradigma dominante. Estamos inmersos en un mundo donde la felicidad se ubica en lo superficial, y la educación forma para la uniformidad. La educación actual, con frecuencia, se abstrae del entorno y no entiende que el placer de aprender no es un placer trivial al que muchas veces se incita por los medios masivos de comunicación, sino un placer propio de la experiencia de vivir, de descubrir, de conocer, de crear; un placer que genera el desarrollo de la confianza y propicia diálogo. Ha desconocido, también, que en situaciones de placer germina la innovación.

La práctica pedagógica actual ha privilegiado la transmisión de informaciones sistematizadas en detrimento de la reinvención de los conocimientos, centrándose, entonces, en la instrucción e ignorando, además, que se aprende con placer y que aprender es un placer.

\section{De la enseñanza al placer de aprender}

La evolución de una visión educativa centrada en la enseñanza que limita su accionar a la transmisión acrítica de informaciones, generalmente descontextualizadas y, por lo tanto, que no 
generan la construcción de sentidos, a una perspectiva de aprendizaje, es realmente un cambio de paradigma que va mucho más allá del ámbito de la didáctica, y se asienta en una epistemología.

La epistemología genética -que inaugura el constructivismo- considera el conocimiento como un proceso de creación; de esta manera el constructivismo supera las posiciones tradicionales de cómo se conoce, a saber, el empirismo que consideraba el conocimiento como una copia de la realidad; y el innatismo que lo creía un despliegue de capacidades que ya posee el organismo. Es así como se puede afirmar que el constructivismo es la antítesis teórica del conductismo y promueve un cambio radical en la pedagogía, trasladando su énfasis de la enseñanza hacia el aprendizaje. No obstante, podemos hablar de tres grandes corrientes dentro del constructivismo: la cognitiva, erigida por Piaget; la socio-histórica, cimentada en Vygotsky, y la biología del amor, establecida por Maturana.

Piaget - considerado el padre del constructivismo- postula que se construye por abstracción reflexiva autorregulada, por equilibración, y que el fin último de la educación es el desarrollo de las operaciones formales, o sea, el pensamiento científico y el juicio moral. Vygotsky, por su parte, expresa que se construye por internalización de la actividad social y, por tanto, el fin de la educación es compartir comunidad de sentido (Citados por Rosas y Sebastián, 2001).

Más recientemente, en la segunda mitad del siglo XX, Humberto Maturana postula una nueva posición respecto a las bases biológicas del conocimiento y plantea que se construye por autopoiesis, por acoplamientos estructurales, así, al determinar que un rasgo característico de los seres vivos es que se producen a sí mismos de manera constante, se entiende que ese dinamismo provoca un permanente cambio estructural. En el marco de esta teoría, se entiende que la estructura cognitiva origina una determinada percepción, lo que significa que el organismo reacciona únicamente frente a un estímulo que es interno al sistema. Dicho en otras palabras, lo que sucede en la interacción no depende únicamente del mensaje sino de la manera como es incorporado a la dinámica autopoiética.

La teoría del conocimiento desarrollada por Maturana cambia la visión hasta ahora aceptada de relación entre sujeto y objeto, pues expresa que el ser vivo se enfrenta al medio como una totalidad circular y que el sistema nervioso opera como una red cerrada de correlaciones neuronales. En palabras del autor, “(...) el encuentro con el medio no rompe esta circularidad, sino que se producen cambios estructurales los que a su vez modifican la deriva de la circularidad". (Maturana y Pörksen, 2004, p. 171). A lo que agrega, "No podemos hablar de nada externo a nuestro vivir y convivir, porque todo lo que hablamos surge en las coordinaciones de coordinaciones de haceres y emociones en nuestro operar en nuestro convivir en el lenguajear" (Maturana y Pörksen, 2004, p. 13).

Para Maturana el fin último de la educación es promover la aceptación del otro como un legítimo otro, y agregaríamos, de la otra como una legítima otra. Estas ideas revolucionan de manera radical la educación y el papel de la educadora o educador, así como de las estudiantes y de los estudiantes, en los procesos pedagógicos y derivan consecuencias que invitan a la innovación.

Dentro de esta nueva visión, el placer constituye una condición primordial para el aprendizaje, pues el miedo y la preocupación obstaculizan los conocimientos, mientras que el placer tiene que ver con lo que se escucha, con la coordinación de coordinaciones.

Las emociones y, particularmente la fruición, forman parte del proceso de conocer. "La emoción de cada momento es lo que modula decisivamente la conducta inteligente" (Maturana y Pörksen, 2004, p. 158). "Los neurocientíficos han encontrado seria evidencia de que la inteligencia, la memoria y las decisiones humanas no son nunca enteramente racionales, sino que siempre están influenciadas por emociones" (Capra, 1998, p. 86). Es más, el aprendizaje es un proceso corporal: aprendemos con todos nuestros sentidos; una atmósfera de tensión genera rigidez en el cuerpo, así 
como lo contrario, un ambiente pedagógico placentero, propicia relajación y situaciones favorables para el aprendizaje.

Assmann (2002) afirma que toda la morfogénesis del conocimiento tiene que ver con la experiencia del placer y que el cerebro-mente está hecho para el gozo del pensar. Entonces, el placer constituye una condición esencial en una educación emergente y, asumir el placer como dinamizador del conocimiento, marca, definitivamente, rumbos ignotos.

\section{Complejidad y belleza en el aprender}

¿Qué se entiende por placer? La definición de placer está en estrecha relación con el modelo de sociedad que se vive. En una sociedad androcrática, el placer se asocia al pecado, al egoísmo y al dolor; se relaciona con el individualismo, el egocentrismo y el consumismo desenfrenado. Por otra parte, desde una visión gilánica, el placer de aprender es fuerza que intensifica la vida; abarca la multidimensionalidad humana, y exhorta al perfeccionamiento constante.

El placer de aprender desvanece las resistencias, relaciona, nos sitúa en los márgenes de las disciplinas, de las perspectivas, de las miradas. Reconocer la complejidad característica de la vida nos lleva a la exploración de alternativas en todos los ámbitos, y requiere la osadía de auto-conocernos. Para ello, la inteligibilidad se queda corta, la ciencia no tiene respuestas. Surge entonces, lo que Wagensberg (1998) llama el principio de la comunicabilidad de complejidades ininteligibles, surge el arte y la cognición estética; el arte como forma de conocimiento que nos accede a un modo distinto de relacionarnos con nosotras mismas, con nosotros mismos, y con el mundo, de representar en una imagen finita la infinitud de la complejidad, y ello es algo grandioso.

"La belleza de un pedazo de realidad es el grado de ritmo y armonía que una mente es capaz de percibir" (Wagensberg, 2004, p. 277). El reto desafiante del aprendizaje, siempre abierto a nuevas posibilidades y al asombro, hace converger en un rizo, placer y belleza. Cuando asumimos la vida con placer y hacemos del aprendizaje una experiencia gozosa, lo que parece imposible nos desafía con la sutileza de la perplejidad, de las posibilidades que ofrece lo desconocido.

Así, la educación y el arte fluyen en el universo de los bordes, se envuelven, se transforman, danzan juntas, crean una nueva dimensión de placer donde el tiempo no transcurre linealmente, sencillamente está.

La educación y el arte crean una dimensión estética, una condición de vida, un modo de cognición a través del cual hacemos mundo (Cerdas, 2008).

\section{Placer y pasión en el aprendizaje}

Se aprende con placer y se aprende con pasión. Placer y pasión son consustanciales a la vida, la pasión desborda los límites, ilumina imposibles, genera fuerza y energía para hacer realidad los sueños.

El placer de aprender induce a entregarse a la creatividad con cada aliento, con la dulzura que da saberse viva o vivo, con la pasión que reclama cada día, porque el placer incita a recrearse en la experiencia universal de existir. Mediante el placer es posible aprovechar el flujo de energía del universo y actuar al unísono, con sincronía, moviéndose en armonía con el cosmos. Una actitud de vivir con gozo permite la magia, propicia alegría, vuelve realidad las quimeras.

Es urgente modificar las percepciones rígidas y despojarse de los lastres de una formación cartesiana y newtoniana para mudar de aires y coadyuvar a que cambie la sociedad. Ese es 
el compromiso que conllevan los principios y valores del nuevo paradigma, para reconocer la borrosidad y la incertidumbre, incorporar lo subjetivo, lo poético y la belleza desde una perspectiva que integre aprendizaje, vida y placer, en un entretejido holístico y complejo.

"La gran tragedia de la sociedad occidental contemporánea es tal vez el hecho de que hayamos perdido la habilidad de experimentar el poder transformador del éxtasis y del gozo" (Johnson, 1992, p. 11). Hoy más que nunca la educación tiene un papel determinante en la formación de personas que aprecien la vida y la convivencia; que aprendan a aprender durante toda su existencia; que se aventuren a ser felices.

Hugo Assmann insiste en que necesitamos volver a introducir en la escuela la experiencia del placer. Nos dice que "(...) cuando está ausente esta dimensión, el aprendizaje se convierte en un proceso meramente instructivo" (Assmann, 2002, p. 28).

Como bien lo señalan Briggs y Peat, “(...) lo imposible es algo que nosotros hacíamos de forma natural cuando éramos niños. Después crecimos dentro de un mundo conceptual más rígido, en el que las fronteras eran absolutas y lo imposible quedó encerrado en un compartimiento separado de lo práctico" (Briggs y Peat, 1999, p. 67). Y, ¿cómo cambiar ese enfoque?, ¿cómo permitirse creer para ver como niñas o niños nuevamente?, ¿cómo aprender a ser feliz?

El gozo es una capacidad intrínseca de las personas que dinamiza los aprendizajes. La búsqueda de alternativas para una educación que supere la visión del paradigma tradicional, fragmentaria y separatista, machista, autoritaria, consumista, que inquiere la certidumbre y promueve la competencia, nos lleva a la necesidad de promover una auténtica participación gozosa donde se integren los intereses, necesidades y visiones de mundo de todas las personas que forman una comunidad. "Una comunidad aprendiente vive inmersa en el placer y el gozo de aprender, tradicionalmente el aprendizaje se asocia con sufrir, en la comunidad no, el aprendizaje es gozoso porque es vivir y la vida en sí misma debe ser gozosa y placentera." (Flores, Flores, Jiménez, Madrigal y Perearnau, 2009, p. 69).

\section{Caminos emergentes, fluir de horizontes}

La educación es un fenómeno social y, por consiguiente, un proceso de transformación de vida en la convivencia; acontece en todas las dimensiones relacionales del vivir en un entrelazamiento de sentimientos, emociones, pensamientos y actuaciones. Bien expresa Maturana que “(...) como las propiedades y características de cada ser vivo están determinadas por su estructura, en la medida que las estructuras de los seres vivos que integran un sistema social cambian, cambian sus propiedades y el sistema social que generan con sus conductas también cambia" (Maturana, 1999, p. 27). Ello nos ubica en una dinámica de tal complejidad que abre múltiples posibilidades y genera retos axiomáticos a la educación. En este sentido, debemos tener presente que, aunque la educación es un instrumento para el mantenimiento y reproducción del sistema dominante, es, también, espacio que puede generar el cambio.

Diversas posibilidades se abren con nuestro caminar; vivimos en un mundo inusitado que nos asombra y conmueve, ante el cual muchas veces, sólo es oportuno el silencio... ‘Quién tiene la capacidad para definir en un momento dado cuáles son las posibilidades reales? ¿Qué ciencia tiene el conocimiento definitivo de lo que es realmente posible?" (Conill, Citado por Chaves, 1999, p. 27). A lo que cabe agregar las palabras de Newton: "Tengo la sensación de haber sido solamente un niño jugando en la playa, y divirtiéndome al encontrar de vez en cuando un guijarro más liso o una concha más bonita de lo habitual, mientras el gran océano de la verdad se extendía ignoto ante mí” (Citado por Kaku, 1998, p. 27).

Aprender es celebrar la vida, así, descubrir lo creativo y amoroso que existe en cada quien y en el mundo, ilumina sendas novedosas, horizontes que propician formas inéditas y gozosas de 
aclamar la vida. Aprender es vivir, vivir es aprender, la vida es la esencia misma de la complejidad, es placer. Entonces, trazar rumbos que incorporen el placer de aprender en la acción educativa, contribuye en la construcción de una nueva etapa en la evolución social, en el tejido del paradigma emergente.

\section{Referencias bibliográficas}

Alvarado, E. (2007). Educación-Ecología-Economía. Pasos hacia otra epistemología. Costa Rica: Alma Mater.

Assmann, H. (2002). Placer y ternura en la educación. Madrid: Narcea.

Briggs, J. y Peat, D. (1999). Las siete leyes del caos. España: Grijalbo.

Capra, F. (1998). La trama de la vida. España: Editorial Anagrama.

Cerdas, J. (2008). Conversatorio Educación y Arte. Costa Rica: Universidad De La Salle.

Chaves, J. (1999). De la utopía a la política económica. España: Editorial San Esteban.

Flores, L., Flores, G., Jiménez, R., Madrigal, J., y Perearnau, M. (2009). Comunidad Aprendiente. Costa Rica: Artemusa Editora.

Gómez, C. (1998). La gestión económica de los recursos naturales y sus críticos. Madrid: Universidad de Alcalá.

Gutiérrez, F. (2008). La educación que tenemos pone en peligro el destino de A. L. Recuperado de http://noticiasdesdeveracruz.blogspot.com/2008/02/dijo-francisco-gutirrez-de-la.html

Johnson, R. (1992). Éxtasis. Psicología del gozo. España: Kairós.

Kaku, M. (1998). Visiones. Cómo la ciencia revolucionará la materia, la vida y la mente en el siglo $X X I$. España: Debate.

Maturana, H. (1999). Transformación en la convivencia. Chile: Dolmen Ediciones.

Maturana, H. y Pörksen, B. (2004). Del ser al hacer. Chile: LOM.

Rosas, R. y Sebastián, C. (2001). Piaget, Vigotski y Maturana. Constructivismo a tres voces. Argentina: AIQUE.

Wagensberg, J. (1998). Ideas sobre la complejidad de mundo. España: Tusquets Editores.

Wagensberg, J. (2004). La rebelión de las formas. España: Tusquets editores. 